\title{
Loewner Matrix Approach for Circuit Modelling in FEM analysis of Microwave Filters
}

\author{
David Young and Valentín de la Rubia
}

\begin{abstract}
A circuit description of the electromagnetic behavior in microwave filters by means of the data provided by FEM analysis in the frequency band of interest is detailed. Loewner matrix approach is used to carry out a tangential interpolation of the simulation data, giving rise to a linear dynamical system description fitting the simulation results. The FEM solver is then used as a black box unlike other model-order reduction techniques. As a result, from this linear dynamical system, a transversal coupling matrix gathering all electromagnetic phenomena within a frequency band is found.

Index Terms-Circuit theory, computer aided engineering, error analysis, finite element methods, Galerkin method, Loewner framework, reduced order systems.
\end{abstract}

\section{INTRODUCTION}

Increasing deployment of telecommunication services is urging microwave industry to speed up product development time, where not only prototyping and final tuning steps in the production line have to be reduced, but also simulation time to achieve an actual electrical design by means of computeraided design (CAD). A significant cost reduction can be achieved in prototyping and tuning steps if electrical designs are optimized to do so, in addition to satisfying electrical specifications. Performing robust electrical designs has thus become of paramount importance, and powerful numerical techniques capable of predicting electromagnetic behaviour are at the heart of this robust design.

Finite Element Methods (FEM) have proven to be accurate in solving the time-harmonic Maxwell's equations for the analysis of microwave filters. However, we still have little knowledge on how electromagnetics actually behaves, and only rather experienced microwave engineers are able to develop high-demanding specification designs. This work focuses on setting up a circuit model description of electromagnetic behavior in microwave filters. As a result, all our expertise as engineers in circuit theory analysis could be used within electromagnetics.

Model order reduction stands upon replacing a rather complex mathematical model by a much simpler approximated one that still maintains certain aspects of the original model [1]. Frequency domain data provided by FEM analysis of microwave filters is processed within the Loewner matrix framework [2], [3], [4] to find a linear dynamical system representation of the physics behind. As a result, the FEM solver is then used as a black box unlike other model-order reduction techniques [5], [6], [7], as it was done in [8].

\section{LOEWNER MATRIX APPROACH}

Let us denote the frequency domain data provided by FEM simulations as $H(s)$, where the complex frequency variable $s$ will be sampled in a specific frequency band. $H\left(s_{i}\right)$ stands for the matrix-valued transfer function sampled at complex frequency $s_{i}$. The goal is to fit a descriptor system state space model of the form

$$
\Sigma:\left\{\begin{array}{rl}
E \frac{d}{d t} x(t) & =A x(t)+B u(t) \\
y(t) & =C x(t)+D u(t)
\end{array} .\right.
$$

$\Sigma$ is a linear dynamical system with $m$-inputs, $m$-outputs and $N$ dimensional internal variable, where $u(t)$ is the input, $y(t)$ is the corresponding output and $x(t)$ is the internal variable. $E, A \in \mathbb{C}^{N \times N}, B \in \mathbb{C}^{N \times m}, C \in \mathbb{C}^{m \times N}$ and $D \in \mathbb{C}^{m \times m}$. $E$ matrix may be singular. The transfer function for the linear dynamical system (1) is

$$
\mathcal{H}(s)=C(s E-A)^{-1} B+D .
$$

The Loewner matrix approach builds a state space model $[E, A, B, C, D]$ up fitting the matrix-valued transfer function data $H\left(s_{i}\right)$ [2], [3].

\section{A. Tangential Interpolation}

Let us take into account the following frequency points in the complex plane $S=\left\{s_{1}, \ldots, s_{p}\right\}$, and its corresponding matrix-valued transfer function evaluations $\left\{H\left(s_{1}\right), \ldots, H\left(s_{p}\right)\right\} . S$ can be divided into two groups

$$
S=\left\{\lambda_{1}, \ldots, \lambda_{k}\right\} \cup\left\{\mu_{1}, \ldots, \mu_{h}\right\} .
$$

The rational interpolation method gives rise to a controllable and observable state space model from the sampled data $H\left(s_{i}\right)$ provided by the FEM analysis of microwave circuits. The tangential interpolation method uses the following right interpolation data

$$
\begin{gathered}
\Lambda=\operatorname{diag}\left\{\lambda_{1}, \ldots, \lambda_{k}\right\} \in \mathbb{C}^{k \times k} \\
R=\left[r_{1}, \ldots, r_{k}\right] \in \mathbb{C}^{m \times k} \\
W=\left[w_{1}, \ldots, w_{k}\right] \in \mathbb{C}^{m \times k}
\end{gathered}
$$

and the left interpolation data

$$
\begin{gathered}
M=\operatorname{diag}\left\{\mu_{1}, \ldots, \mu_{h}\right\} \in \mathbb{C}^{h \times h} \\
L=\left[\begin{array}{c}
l_{1} \\
\vdots \\
l_{h}
\end{array}\right] \in \mathbb{C}^{h \times m}, V=\left[\begin{array}{c}
v_{1} \\
\vdots \\
v_{h}
\end{array}\right] \in \mathbb{C}^{h \times m} .
\end{gathered}
$$


$r_{i}$ and $l_{j}$ stand for right and left tangential directions respectively. The tangential interpolation method sets a minimal dynamical system realization $[E, A, B, C, D]$ up such that the corresponding transfer function $\mathcal{H}(s)$ satisfies the following right and left constraints

$$
\begin{aligned}
& \mathcal{H}\left(\lambda_{i}\right) r_{i}=H\left(\lambda_{i}\right) r_{i}=w_{i} \\
& l_{j} \mathcal{H}\left(\mu_{j}\right)=l_{j} H\left(\mu_{j}\right)=v_{j}
\end{aligned}
$$

Loewner matrix and shifted Loewner matrix are specially well-suited to face this tangential interpolation problem since these matrices, by construction, give rise to a system realization fitting the simulation data [2]. The Loewner matrix of $H(s)$ can be built as

$$
\mathcal{L}=\left(\begin{array}{ccc}
\frac{v_{1} r_{1}-l_{1} w_{1}}{\mu_{1}-\lambda_{1}} & \ldots & \frac{v_{1} r_{k}-l_{1} w_{k}}{\mu_{1}-\lambda_{k}} \\
\vdots & \ddots & \vdots \\
\frac{v_{h} r_{1}-l_{h} w_{1}}{\mu_{h}-\lambda_{1}} & \ldots & \frac{v_{h} r_{k}-l_{h} w_{k}}{\mu_{h}-\lambda_{k}}
\end{array}\right)
$$

Similarly, the shifted Loewner matrix can be obtained by setting the Loewner matrix of $s H(s)$

$$
\sigma \mathcal{L}=\left(\begin{array}{ccc}
\frac{\mu_{1} v_{1} r_{1}-\lambda_{1} l_{1} w_{1}}{\mu_{1}-\lambda_{1}} & \ldots & \frac{\mu_{1} v_{1} r_{k}-\lambda_{k} l_{1} w_{k}}{\mu_{1}-\lambda_{k}} \\
\vdots & \ddots & \vdots \\
\frac{\mu_{h} v_{h} r_{1}-\lambda_{1} l_{h} w_{1}}{\mu_{h}-\lambda_{1}} & \ldots & \frac{\mu_{h} v_{h} r_{k}-\lambda_{k} l_{h} w_{k}}{\mu_{h}-\lambda_{k}}
\end{array}\right)
$$

Finally, recalling fundamental results from Loewner framework, cf. [2], the function

$$
H(s)=(W-D R)((\sigma \mathcal{L}-L D R)-s \mathcal{L})^{-1}(V-L D)+D
$$

interpolates the simulation data.

\section{B. Circuit Model}

Equation (9) not only allows us to compute the frequency response in the microwave circuit in a straightforward way, but also, further manipulations can be carried out to get further insight of the electromagnetic problem. Since tangential interpolation strategy is taken into account, the data-driven reduced-order model obtained is a surrogate of the Maxwell problem in the frequency band of interest. Diagonalization of the Loewner and shifted Loewner Matrix pencil gives rise to a circuit representation in (9) where global eigenresonances are taken into account as internal space state variables. The rational transfer function is then factored out as a partial fraction expansion. This matrix respresentation gives rise to the transversal coupling matrix introduced by Cameron [9]. As a result, we have a circuit representation of the electromagnetic behaviour by means of simulation data for the microwave filter under analysis. Since transversal coupling matrix is obtained, similarity transformations can be applied to get the coupling topology in the actual microwave filter layout. This serves for filter design and diagnosis purposes as all electromagnetic phenomena are taking into account.

$\left(\begin{array}{cccccccccccc}0 & 1.0 & 0 & 0 & 0 & 0 & 0 & 0 & 0 & 0 & 0 & 0 \\ 1.0 & -0.4 & 0.8 & 0 & 0 & 0 & 0 & 0 & 0 & 0 & 0 & 0 \\ 0 & 0.8 & 0.0 & 0.6 & 0 & 0 & 0 & 0 & 0 & 0 & 0 & 0 \\ 0 & 0 & 0.6 & \lambda_{3} & 0.5 & 0.2 & 0 & 0 & 0 & 0 & 0 & 0 \\ 0 & 0 & 0 & 0.5 & -0.4 & 0.5 & 0 & 0 & 0 & 0 & 0 & 0 \\ 0 & 0 & 0 & 0.2 & 0.5 & 0.0 & 0.5 & 0 & 0 & 0 & 0 & 0 \\ 0 & 0 & 0 & 0 & 0 & 0.5 & 0.0 & 0.5 & 0.2 & 0 & 0 & 0.0 \\ 0 & 0 & 0 & 0 & 0 & 0 & 0.5 & -0.5 & 0.5 & 0 & 0 & 0.0 \\ 0 & 0 & 0 & 0 & 0 & 0 & 0.2 & 0.5 & 0.0 & 0.6 & 0 & 0.0 \\ 0 & 0 & 0 & 0 & 0 & 0 & 0 & 0 & 0.6 & 0.2 & 0.8 & 0.2 \\ 0 & 0 & 0 & 0 & 0 & 0 & 0 & 0 & 0 & 0.8 & -0.4 & 1.0 \\ 0 & 0 & 0 & 0 & 0 & 0 & 0.0 & 0.0 & 0.0 & 0.2 & 1.0 & 0\end{array}\right)$

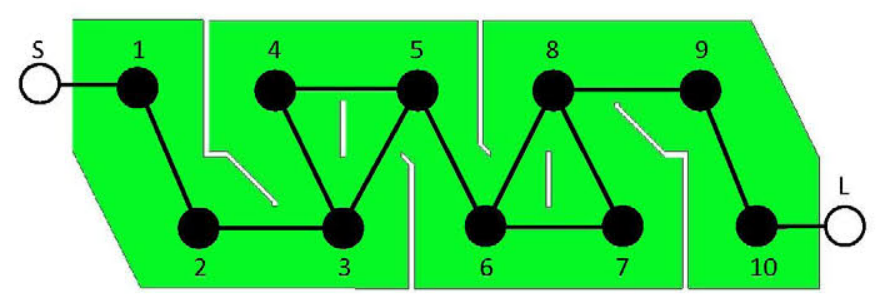

Fig. $1.10^{\text {th }}$ order $1800 \mathrm{MHz}$ filter.

\section{NUMERICAL RESULTS}

In this section, we apply the proposed Loewner approach circuit modelling for diagnosis in an $10^{\text {th }}$ order $1800 \mathrm{MHz}$ filter with two transmission zeros. See Fig. 1. The 1.6-1.9 $\mathrm{GHz}$ band is taken into account. In this case, a data-driven reduced-order model of dimension 13 is obtained where 10 eigenmodes correspond to dominant global resonances, which is the order of the filter, and the remaining 3 eigenmodes are higher order resonances standing for parasitics behaviour in electromagnetics. Higher order resonances are taken into account as direct source to load coupling in the transversal coupling matrix. After applying a narrow band approximation to the frequency dependent transversal coupling matrix in order to obtain a coupling matrix with constant coefficients, cf. [10], we monitor the tuning in resonator 3 in Fig 1. Equation (10) shows the coupling matrix results when this tuning is done. Only parameter $\lambda_{3}$ is noticed to change during this process. These results agree with the expected behaviour.

\section{CONCLUSION}

A data-driven reduced-order model in FEM analysis for microwave filters has been detailed. The Loewner matrix approach has been used for tangential interpolation of the simulation data. A minimal dynamical system realization has been shown and special emphasis has been placed in describing this dynamical system as a circuit, such that the electromagnetic behavior in microwave filters is written down in terms of simple coupling matrix theory in the frequency band of interest.

\section{REFERENCES}

[1] A. C. Antoulas, Approximation of Large-Scale Dynamical Systems. Philadelphia, PA: SIAM, 2005. 
[2] A. J. Mayo and A. C. Antoulas, "A framework for the solution of the generalized realization problem," Linear Algebra and its Applications, no. 425 , pp. 634 662, Aug. 2007.

[3] S. Lefteriu and A. C. Antoulas, "A new approach to modeling multiport systems from frequency-domain data," IEEE Trans. Computer-Aided Design, vol. 29, no. 1, pp. 14-27, Jan. 2010.

[4] G. Gurrala, "Loewner matrix approach for modelling FDNE of power systems," Electric Power Systems Research, vol. 125, pp. 116-123, 2015.

[5] V. de la Rubia, U. Razafison, and Y. Maday, "Reliable fast frequency sweep for microwave devices via the reduced-basis method," IEEE Trans. Microwave Theory Tech., vol. 57, no. 12, pp. 2923-2937, Dec. 2009.

[6] A. Sommer, O. Farle, and R. Dyczij-Edlinger, "A new method for accurate and efficient residual computation in adaptive model-order reduction," IEEE Trans. Magn., vol. 51, no. 3, pp. 1-4, Mar. 2015.

[7] G. Fotyga, K. Nyka, and M. Mrozowski, "Multilevel model order reduction with generalized compression of boundaries for 3-d FEM electromagnetic analysis," Progress In Electromagnetics Research, vol. 139, pp. 743-759, 2013.

[8] V. de la Rubia, D. Young, and D. Liang, "Circuit model for microwave filters via the reduced-basis method," in 2016 IEEE MTT-S International Conference on Numerical Electromagnetic and Multiphysics Modeling and Optimization (NEMO), July 2016, pp. 1-2.

[9] R. J. Cameron, "Advanced coupling matrix synthesis techniques for microwave filters," IEEE Trans. Microwave Theory Tech., vol. 51, no. 1, pp. 1-10, Jan. 2003.

[10] S. Amari and M. Bekheit, "Physical interpretation and implications of similarity transformations in coupled resonator filter design," IEEE Trans. Microwave Theory Tech., vol. 55, no. 6, pp. 1139-1153, Jun. 2007. 